\title{
Risk Factors and Etiologies of Ischemic Strokes in Young Patients: A Tertiary Hospital Study in North India
}

\author{
Deepa Dash, Ashu Bhashin, Awadh kumar Pandit, Manjari Tripathi, Rohit bhatia, Kameshwar Prasad, \\ Madakasira Vasantha Padma \\ Department of Neurology, All India Institute of Medical Sciences, Delhi, India
}

Background and Purpose Stroke in young adults has a special significance in developing countries, as it affects the most economically productive group of the society. We identified the risk factors and etiologies of young patients who suffered ischemic strokes and were admitted to a tertiary referral hospital in North India.

Methods A retrospective review of case records from patients with ischemic stroke in the age range of 18-45 years was conducted from 2005 to 2010. Data regarding patients' clinical profiles, medical histories, diagnostic test results, and modified Rankin Scale scores at hospital discharge were examined. Stroke subtyping was conducted in accordance with the Trial of Org 10172 in Acute Stroke Treatment (TOAST) criteria.

Results Of the 2,634 patients admitted for ischemic stroke, 440 (16.7\%) were in the 18-45 year age range and the majority (83.4\%) were male. The most common risk factors were hypertension (34.4\%) and dyslipidemia (26.5\%). The most common subtype of stroke was undetermined (57\%), followed by other determined causes (17.3\%). Among the category of undetermined etiology, incomplete evaluation was the most common. Most of the patients demonstrated good functional outcomes.

Conclusions Young adults account for $16.7 \%$ of all stroke patients in North India. Risk factors are relatively prevalent, and a high proportion of the patients are categorized under undetermined and other determined causes. The results highlight the needs for aggressive management of traditional risk factors and extensive patient work-ups to identify stroke etiology in India.
Correspondence: Madakasira Vasantha Padma

Department of Neurology, Room No 708, Neurosciences Centre, All India Institute of Medical Sciences, New Delhi 110029, India

Tel: +91-26594794, 26588700/26589900 Ext: 4794

E-mail:vasanthapadma123@gmail.com

Received: January 27, 2014

Revised: August 5, 2014

Accepted: August 6, 2014

The authors have no financial conflicts of interest.

Keywords Young adult; Ischemic stroke; India; TOAST classification

\section{Introduction}

Ischemic strokes in young adults can have a significant impact on the affected individuals, their families, and societies in general, as the patients are affected in the economically productive period of their lives. Almost two thirds of the global burden of stroke is borne by those in developing countries. ${ }^{1}$ Young adults account for approximately $10 \%-30 \%$ of all stroke patients in India, as opposed to $3 \%-8.5 \%$ in Western countries. ${ }^{2.4}$ The etiolo- gies of ischemic strokes in young adults are different and more diverse as compared to those observed in the elderly. Furthermore, etiological subtyping also varies according to geographical distribution. It is important to identify the causative factors in young stroke patients in order to prevent recurrences appropriately. Despite its substantial societal impact, there remains a paucity of literature regarding the etiological subtyping and risk factors for ischemic stroke in young Indian patients. The current study was conducted to identify ischemic stroke risk fac- 
tors and etiological subtypes according to the Trial of Org 10172 in Acute Stroke Treatment (TOAST) criteria in young Indian patients. $^{5}$

\section{Methods}

We conducted a retrospective chart review of patients who suffered an ischemic stroke from January 2005 to December 2010 and were admitted to a tertiary care hospital in North India. Ischemic stroke was defined as a sudden focal neurologic deficit with imaging-confirmed cerebral infarction. We excluded cases of intracranial hemorrhage, subarachnoid hemorrhage, and cerebral venous thrombosis.

From the total population of ischemic stroke patients, patients who had suffered an ischemic stroke and were in the age range of 18-45 years were enrolled. As a matter of protocol, all stroke patients admitted to the neurology wards underwent routine hematological and biochemistry tests (complete blood count, prothrombin time, activated partial thromboplastin time reactive protein, serum creatinine, blood glucose and lipid profile), chest X-rays, electrocardiography, and brain imaging including computed tomography (CT) and/or magnetic resonance imaging (MRI) at admission. Additionally, a majority of patients underwent vascular imaging studies such as MR angiography or CT angiography. Transthoracic echocardiography and Holter monitoring were performed in selected patients. More specific diagnostic testing (e.g. vasculitis profile) was performed when deemed necessary by the treating neurologist.

Patient case records were reviewed by a neurologist (AKP) and data regarding each patient's demographic profile, clinical presentation, medical history (emphasis on risk factors), results of vascular imaging, biochemical profile and other diagnostic tests, in-hospital treatment, and the modified Rankin Scale (mRS) score at hospital discharge were recorded in a structured proforma. Stroke subtyping was based on the information available at discharge and according to the TOAST criteria. Diabetes mellitus, hypertension, smoking, dyslipidemia, previous stroke or transient ischemic attack, migraine, atrial fibrillation, coronary artery disease, alcohol consumption, drug abuse, and family history of stroke among first and second-degree relatives were considered as risk factors. Etiological classification was analyzed by dividing the cohort into 2 groups according to age (18-35 years and $36-45$ years) and by gender.

The recorded data were statistically analyzed for the percentage and mean and standard deviation of all variables. SPSS version 16.0 for windows was used for all statistical analyses. The $\chi^{2}$, Fisher's exact, and $t$ tests were used as appropriate. $P<0.05$ was considered as statistically significant.

\section{Results}

Of 2,634 ischemic stroke patients admitted to our institute from January 2005 to December 2010, 440 patients were in the range of 18-45 years. The male-to-female ratio was 5:1, and the mean patient age was $38.9 \pm 7.1$ years. Identified risk factors for ischemic stroke were hypertension (44.5\%), dyslipidemia (26.1\%), prior stroke or transient ischemic attack (TIA; 26\%), family history of stroke (15.7\%), and diabetes (13.9\%) (Table 1). All patients underwent CT scans and 38.4\% underwent MR angiography. Additionally, a carotid artery duplex scan was conducted in $85.7 \%$ of the patients. Holter monitoring was performed in $63 \%$ of patients, transthoracic echocardiography in $96.3 \%$, and transesophageal echocardiography in $2.3 \%$. A detailed workup for coagulation disorders was conducted in $32 \%$ of the patients.

The anterior circulation was the most commonly involved vascular territory $(74.2 \%)$, followed by the posterior circulation (21.1\%), and strokes involving both territories (4.7\%). The most common TOAST subtype was undetermined (57\%), followed by other determined cause (ODC; 17.3\%), and cardioembolism (14\%) (Table 1). The etiological classification was also analyzed by dividing the cohort into 2 groups according to age (18-35 years and $36-45$ years). The $36-45$ year age group had a significantly higher percentage of patients with cardioembolic stroke (Table 2). When data were analyzed according to gender, the mean ages of male and females patients were $39.5 \pm 7.1$

Table 1. Risk factors and demographic characteristics

\begin{tabular}{lc}
\hline Risk factors & $\mathrm{n}=440$ \\
\hline Alcohol & $42(9.5 \%)$ \\
Smoking & $42(9.5 \%)$ \\
Prior stroke & $117(26 . \%)$ \\
Family history of cerebrovascular disease & $69(15.7 \%)$ \\
Hypertension & $196(44.5 \%)$ \\
Diabetes & $61(13.9 \%)$ \\
Dyslipidemia & $115(26.1 \%)$ \\
Illicit drug use & $44(10 \%)$ \\
Valvular heart disease & $56(12.7 \%)$ \\
Cardiomyopathy & $12(2.7 \%)$ \\
Atrial fibrillation & $29(6.6 \%)$ \\
Coronary artery disease & $24(5.45 \%)$ \\
\hline Toast subtype & \\
\hline Large artery atherosclerosis & $21(4.7 \%)$ \\
Cardio-embolic & $62(14 \%)$ \\
Small artery disease & $30(6.8 \%)$ \\
Other determined causes & $76(17.3 \%)$ \\
Undetermined- Total & $251(57 \%)$ \\
Incomplete evaluation & $243(96.8 \%)$ \\
Complete evaluation: No etiology found & $1(0.3 \%)$ \\
More than two etiologies & $7(2.7 \%)$ \\
\hline
\end{tabular}


Table 2. TOAST classification according to age group

\begin{tabular}{lccl}
\hline Subtypes & $\begin{array}{c}18-35 \text { years } \\
n=132(28 \%)\end{array}$ & $\begin{array}{c}36-45 \text { years } \\
n=308(72 \%)\end{array}$ & Pvalue \\
\hline Large artery & $6(4.5 \%)$ & $15(4.9 \%)$ & 1.00 \\
Cardio-embolic & $4(3 \%)$ & $58(18.8 \%)$ & 0.0001 \\
Small vessel disease & $11(8.3 \%)$ & $19(6.2 \%)$ & 0.41 \\
Other determined causes & $23(17.4 \%)$ & $53(17.0 \%)$ & 0.78 \\
Undetermined cause & $88(66.6 \%)$ & $163(52.9 \%)$ & 0.008 \\
$\quad$ Incomplete evaluation & 85 & 158 & \\
$\quad$ Complete evaluation & 1 & 0 & \\
Multiple etiologies & 2 & 5 & \\
\hline
\end{tabular}

Table 3. TOAST classification according to gender

\begin{tabular}{lccc}
\hline Subtypes & Male $(\mathrm{n}=367)$ & Female $(\mathrm{n}=73)$ & $P$ value \\
\hline Large artery & $18(4.9 \%)$ & $3(4.1 \%)$ & 0.771 \\
Cardio-embolic & $40(10.8 \%)$ & $22(30.1 \%)$ & 0.000 \\
Small artery disease & $20(5.44 \%)$ & $10(13.69 \%)$ & 0.011 \\
Other determined Causes & $57(15.5 \%)$ & $19(26.0 \%)$ & 0.030 \\
Undetermined cause & $232(63.2 \%)$ & $19(26.0 \%)$ & 0.001 \\
\hline
\end{tabular}

and $36.0 \pm 6.7$ years, respectively $(P=0.730)$. Women were more likely than men to have cardioembolism, small artery disease, and ODC (Tables 3, 4).

There were 76 patients who had ODC as an etiological subtype. The most common etiology in this group was dissection (Table 5). Among 39 cases of dissection, there were 37 patients with internal carotid artery dissection and 2 patients with vertebral artery dissection. The most common cause of cardioembolic stroke was valvular heart disease associated with rheumatic heart disease (44 patients, 70.1\%). Cardiomyopathy was found in 4 (6\%) patients, atrial fibrillation in $4(6 \%)$, and others (i.e. infective endocarditis in 4 and myocardial infarction in 6) in 16.1\%.

Regarding treatment, thrombolysis was performed in $3.9 \%$ of patients. Most (84\%) of the patients were on an anti-platelet treatment with a majority $(65.4 \%)$ receiving aspirin alone. Almost $88 \%$ of patients with a prior history of stroke and TIA had not taken any medications for the secondary prevention of stroke. At the time of hospital discharge, 392 (89\%) patients had mRS scores in the range of $0-2$, and $37(8.4 \%)$ patients hand $\mathrm{mRS}$ scores of 3 or 4 . Death was reported in $11(2.5 \%)$ patients.

\section{Discussion}

By 2050 , it is anticipated that $80 \%$ of stroke patients will be from the developing regions of the world. ${ }^{6}$ Additionally, there is growing evidence for an increasing trend in the incidence of stroke in young adults. ${ }^{7}$ Accurate classification of ischemic stroke is critical for guiding treatment decisions and determining the prognosis of individual patients. However, few studies have fo-
Table 4. Risk factors according to gender

\begin{tabular}{lccc}
\hline Subtypes & Male $(\mathrm{n}=367)$ & Female $(\mathrm{n}=73)$ & $P$ value \\
\hline Alcohol & $42(11.4 \%)$ & 0 & 0.000 \\
Smoking & $40(10.9 \%)$ & $2(2.7 \%)$ & 0.000 \\
Prior stroke & $101(27.5 \%)$ & $16(2.2 \%)$ & 0.322 \\
Family history of cerebrovas- & $67(18.2 \%)$ & $2(2.7 \%)$ & 0.001 \\
$\quad$ cular disease & & & \\
Hypertension & $175(47.7 \%)$ & $21(28.7 \%)$ & 0.003 \\
Diabetes & $49(1.3 \%)$ & $12(16.4 \%)$ & 0.486 \\
Dyslipidemia & $99(26.9 \%)$ & $16(21.9 \%)$ & 0.369 \\
Illicit drug use & $44(11.9 \%)$ & 0 & 0.000 \\
Valvular heart disease & $34(9.2 \%)$ & $22(30.1 \%)$ & 0.000 \\
Cardiomyopathy & $7(1.9 \%)$ & $5(6.8 \%)$ & 0.018 \\
Atrial fibrillation & $17(4.6 \%)$ & $12(16.4 \%)$ & 0.000 \\
Coronary artery disease & $24(6.5 \%)$ & 0 & 0.000
\end{tabular}

Table 5. Etiologies under other determined causes

\begin{tabular}{lc}
\hline Etiology & $\mathrm{n}=76$ \\
\hline Dissection & $39(51.3 \%)$ \\
Infectious arteritis (tuberculosis) & $7(9.2 \%)$ \\
Hyperhomocysteinemia & $7(9.2 \%)$ \\
RCVS & $6(7.8 \%)$ \\
Migraine related & $4(5.2 \%)$ \\
Plasmacytoma-related & $4(5.2 \%)$ \\
Raised lipoprotein A & $3(3.9 \%)$ \\
Moyamoya & $2(2.6 \%)$ \\
Chronic idiopathic thrombocytopenic purpura & $2(2.6 \%)$ \\
Cancer & $2(2.6 \%)$ \\
\hline
\end{tabular}

cused on the etiology and risk factors for ischemic stroke in young adults in India. ${ }^{8-10}$ The current study is the largest one focusing young stroke patients in India.

In comparison to studies conducted in Western countries, a higher male predominance was observed in the current study. ${ }^{11}$ The percentage was even higher than that reported from earlier studies from India. ${ }^{8,9}$ Because our institute is a referral center, patients are referred from all over the country. The male predominance noted in the current study may at least in part have been attributable to a sociocultural bias in India that manifests as males being more likely to seek treatment at referral centers than females. Similar to the previously published results, ${ }^{12,13}$ the current study identified a relatively high frequency of modifiable risk factors for ischemic stroke. Of these, hypertension and dyslipidemia were the most common. A significant number of patients had a history of prior stroke and TIA, and almost $88 \%$ of them were not taking any medications for the secondary prevention of stroke. This observation emphasizes the need for aggressive primary and secondary prevention targeting the traditional modifiable risk factors for young Indian subjects.

The most common stroke subtype in the current study was undetermined cause, and was observed at a higher rate than that 
reported in previous studies of ischemic stroke in young adults (24\%-36\%). ${ }^{12,14-17}$ The most frequent reason for categorizing these patients as having an undetermined etiology was an incomplete evaluation. Most of the patients did not have insurance and were therefore unable to afford the costs of radiological and biochemical diagnostic tests. The second most common etiology in the current study was stroke due to ODC. This result was comparable to the results of previous studies from Western countries. ${ }^{18-20}$

In a case controlled study of 214 first-occurrence ischemic strokes in young adults from South India, Lipska et al. ${ }^{9}$ reported that cardioembolic stroke (25.2\%) was the most common stroke subtype, followed by large artery atherosclerosis (12.6\%). They reported that $11.2 \%$ of patients had ODC. In another retrospective study of 177 young adult ischemic stroke patients from South India, the most common etiologic subtype was atherothrombotic stroke (24\%), followed by cardioembolic stroke (17\%). ${ }^{8}$ In contrast to the results of the current study, the aforementioned studies reported a low proportion of patients with ODC. This difference may be attributable to the non-availability of newer imaging techniques and diagnostic facilities in the previous studies, which could have resulted in an underreporting of ODC. In our study, the most common etiology under the category of ODC was cerebral artery dissections, which accounted for $51.3 \%$ of cases. The prevalence of cerebral artery dissection in young stroke patients has been reported to be in the range of $10 \%-20 \%{ }^{21}$ Similarly, $8.8 \%$ of patients in the current study were found to have dissections. Therefore, careful evaluation for cerebral artery dissection is pertinent in all young ischemic stroke patients. Detailed vascular imaging would also further increase the detection rates of other rare causes of stroke in young adults, such as Moyamoya disease and other vasculopathies.

The most common cause of cardioembolic stroke in the current study was valvular heart disease, with the majority having rheumatic heart disease (RHD). The incidence of cardioembolic stroke in young adults ranges from $14 \%$ to $47 \%{ }^{11,22-24}$ In the current study, cardioembolic stroke accounted for $14 \%$ of patients. Previous studies from India have reported RHD and prosthetic valves contributing to almost two thirds of all cardioembolic strokes in young adults. ${ }^{8,9}$ This highlights the need to develop preventive strategies targeting patients with RHD in order to ensure proper anticoagulation and medical follow-up for the disease. This is in contrast to the results of studies from developed countries where etiologies such as patent foramen ovale (PFO) or atrial septal defects (ASD) were more common than valvular heart disease as a cause of stroke. ${ }^{11,26}$ Another reason for underestimation of PFO/ASD could be the very low rate of transesophageal echocardiography performance (2.3\%) in the current study. We found that a significantly higher percentage of the cardioembolic subtype was found in women, which is consistent with previous studies that reported a higher prevalence rate of RHD in women. ${ }^{27,28}$

In the current study, only $6.8 \%$ of patients were categorized as having small-vessel disease. TOAST defines small-vessel disease only if the subcortical lesions are smaller than $15 \mathrm{~mm}$ and large artery atherosclerosis only if there is stenosis $(>50 \%)$ in the corresponding artery. ${ }^{5}$ Therefore, subcortical infarction of $>15$ $\mathrm{mm}$ in size and infarctions with vulnerable plaque with stenosis $<50 \%$ should be classified as stroke of undetermined etiology. Thus, we may need a newer subtype classification in order to clarify these cases more accurately. ${ }^{29}$ The proportion of young patients with large artery atherosclerosis has traditionally been low in studies from Western countries. ${ }^{30,31}$ Despite the high prevalence of the traditional risk factors for stroke in our cohort, only $4.7 \%$ of patients were found to have large artery disease. This could be due to the fact that only $38.4 \%$ of our patients underwent intracranial vessel imaging.

In a prospective study of 198 young ischemic stroke patients, Nedeltchev and colleagues reported that $68 \%$ of patients achieved an $\mathrm{mRS}$ score of $0-1,26 \%$ had an $\mathrm{mRS}$ score of $2-5$, and $3 \%$ had died within 3 months. ${ }^{14}$ Putaala et al. found a functionally independent outcome ( $\mathrm{mRS}$ score $=0-2$ ) in $80 \%$ of patients in a prospective study of 968 patients. ${ }^{31}$ In line with these results, good functional outcomes at discharge $(\mathrm{mRS}$ range $=0-2)$ were observed in $89 \%$ of patients in our cohort. These results suggest relatively good functional outcomes in young stroke patients both in western countries and in India.

The current study has several limitations, the first being the retrospective design. Because patients were recruited over a long period of time, differences in the work-up strategy with regard to advanced imaging and other diagnostic technologies may have been introduced. Furthermore, not all patients underwent detailed studies such as tests for coagulation abnormalities, vascular imaging, and transesophageal echocardiography. Lastly, this is a hospital-based study and the results may not be generalizable to the general population. Despite these limitations, the current study is the largest one focusing on the etiologies and risk factors for ischemic stroke in young adults in India. Our study highlights the need for aggressive management of traditional risk factors in young adults and also the need for extensive work-up in every patient in order to find correct etiologies.

\section{References}

1. Bonita R, Mendis S, Truelsen T, Bogousslavsky J, Toole J, Yatsu F. The global stroke initiative. Lancet Neurol 2004;3:391-393. 
2. Kaul S, Bandaru VC, Suvarna A, Boddu DB. Stroke burden and risk factors in developing countries with special reference to India. J Indian Med Assoc 2009; 107:358-370.

3. Nagaraja D, Gururaj G, Girish N, Panda S, Roy AK, Sarma GR, et al. Feasibility study of stroke surveillance: data from Bangalore, India. Indian J Med Res 2009;130:396-403.

4. Nencini P, Inzitari D, Baruffi MC, Fratiglioni L, Gagliardi R, Benvenuti L, et al. Incidence of stroke in young adults in Florence, Italy. Stroke 1988:19:977-998.

5. Adams HP Jr, Bendixen BH, Kappelle LJ, Biller J, Love BB, Gordon DL, et al. Classification of subtype of acute ischemic stroke: definitions for use in a multicenter clinical trial. Stroke 1993;24: 35-41.

6. Feigin VL. Stroke epidemiology in the developing world. Lancet 2005;365:2160-2161.

7. George MG, Tong X, Kuklina EV, Kuklina EV, Labarthe DR. Trends in stroke hospitalizations and associated risk factors among children and young adults, 1995-2008. Ann Neurol 2011; 70:713-721.

8. Nayak SD, Nair M, Radhakrishnan K, Sarma PS. Ischemic stroke in the young adult: clinical features, risk factors and outcome. Natl Med J India 1997;10:107-112.

9. Lipska K, Sylaja PN, Sarma PS, Thankappan KR, Kutty VR, Vasan RS, et al. Risk factors for acute ischaemic stroke in young adults in South India. J Neurol Neurosurg Psychiatry 2007;78: 959-963.

10. Mehndiratta MM, Agarwal P, Sen K, Sharma B. Stroke in young adults: a study from a university hospital in north India. Med Sci Monit 2004;10:CR535-CR541.

11. Ji R, Schwamm LH, Pervez MA, Singhal AB. Ischemic stroke and transient ischemic attack in young adults: risk factors, diagnostic yield, neuroimaging, and thrombolysis. JAMA Neurol 2013;70:51-57.

12. You RX, McNeil JJ, O’Malley HM, Davis SM, Thrift AG, Donnan GA. Risk factors for stroke due to cerebral infarction in young adults. Stroke 1997;28:1913-1918.

13. Rohr J, Kittner S, Feeser B, Hebel JR, Whyte MG, Weinstein A, et al. Traditional risk factors and ischemic stroke in young adults: the Baltimore-Washington Cooperative Young Stroke Study. Arch Neurol 1996;53:603-607.

14. Nedeltchev K, der Maur TA, Georgiadis D, Arnold M, Caso V, Mattle HP, et al. Ischemic stroke in young adults: predictors of outcome and recurrence. J Neurol Neurosurg Psychiatry 2005; 76:191-195.

15. Varona JF, Guerra JM, Bermejo F, Molina JA, Gomez de la Camara, A. Causes of ischemic stroke in young adults, and evolution of the etiological diagnosis over the long term. Eur Neurol 2007;57:212-218.
16. Kittner SJ, Stern BJ, Wozniak M, Buchholz DW, Earley CJ, Feeser BR, et al. Cerebral infarction in young adults: the BaltimoreWashington Cooperative Young Stroke Study. Neurology 1998; 50:890-894.

17. Rasura M, Spalloni A, Ferrari M, De Castro S, Patella R, Lisi F, et al. A case series of young stroke in Rome. Eur J Neurol 2006; 13:146-152.

18. Cerrato P, Grasso M, Imperiale D, Priano L, Baima C, Giraudo $\mathrm{M}$, et al. Stroke in young patients: etiopathogenesis and risk factors in different age classes. Cerebrovasc Dis 2004,18:154-159.

19. Ghandehari K, Moud ZI. Incidence and etiology of ischemic stroke in Persian young adults. Acta Neurol Scand 2006,113: 121-124.

20. Groppo E, De Gennaro R, Granieri G, Fazio P, Cesnik E, Granieri $\mathrm{E}$, et al. Incidence and prognosis of stroke in young adults: a population-based study in Ferrara, Italy. Neurol Sci 2012;33: 53-58.

21. Schievink WI. Spontaneous dissection of the carotid and vertebral arteries. N Engl J Med 2001;344:898-906.

22. Chan MT, Nadareishvili ZG, Norris JW. Diagnostic strategies in young patients with ischemic stroke in Canada. Can J Neurol Sci 2000;27:120-124.

23. Ferro JM, Crespo M. Prognosis after transient ischemic attack and ischemic stroke in young adults. Stroke 1994;25:1611-1616.

24. Yesilot Barlas N, Putaala J, Waje-Andreassen U, Vassilopoulou S, Nardi K, Odier C, et al. Etiology of first-ever ischaemic stroke in European young adults: the 15 cities young stroke study. Eur J Neurol 2013;20:1431-1439.

25. Siqueira Neto JI, Santos AC, Cabete Fabio SR, Sakamoto AC. Cerebral infarction in patients aged 15 to 40 years. Stroke 1996; 27:2016-2019.

26. Putaala J, Metso AJ, Konkola N, Kraemer Y, Haapaniemi E, Kaste M, et al. Analysis of 1,008 consecutive patients aged 15 to 49 with first-ever ischemic stroke: the Helsinki young stroke registry. Stroke 2009;40:1195-1203.

27. Kim BJ, Kim JS. Ischemic stroke subtype classification: an Asian view point. J Stroke 2014:16:8-17.

28. Bogousslavsky J, Pierre P. Ischemic stroke in patients under age 45. Neurol Clin 1992;10:113-124.

29. Leys D, Bandu L, Hénon H, Lucas C, Mounier-Vehier F, Rondepierre $\mathrm{P}$, et al. Clinical outcome in 287 consecutive young adults (15 to 45 years) with ischemic stroke. Neurology 2002;59:26-33.

30. Siqueira Neto JI, Santos AC, Fabio SR, Sakamoto AC. Cerebral infarction in patients aged 15 to 40 years. Stroke 1996;27: 2016-2019.

31. Putaala J, Strbian D, Mustanoja S, Haapaniemi E, Kaste M, Tatlisumak T. Functional outcome in young adult ischemic stroke: impact of lipoproteins. Acta Neurol Scand 2013;127:61-69. 